\title{
High fitness is associated with a better cardiovascular risk profile in patients with type 2 diabetes mellitus
}

\author{
Claudia RL Cardoso ${ }^{1}$, Maicon DL Maia ${ }^{2}$, Fátima P de Oliveira $^{2}$, Nathalie C Leite ${ }^{1}$ and Gil F Salles ${ }^{1}$
}

Novel cardiovascular risk markers, such as ambulatory blood pressure (BP) and aortic stiffness, have been proposed. The aim of this study was to investigate the relationship between physical fitness and some of these risk markers in $\mathbf{5 7 5}$ type 2 diabetic patients. In a cross-sectional design, clinical, laboratory, echocardiographic, aortic pulse wave velocity and 24-h ambulatory BP monitoring data were obtained. Fitness was self-reported using a standard questionnaire of daily activities, and then assessed as belonging to one of the three categories: low ( $<4$ metabolic equivalents (METs)), moderate ( $\geqslant 4$ to $<7$ METs) and high fitness ( $\geqslant 7$ METs). In a random sub-sample of 265 patients, self-reported fitness was confirmed by a standard treadmill test. Statistical analysis was carried out, using tests including bivariate tests among the three categories and multivariate logistic regression. Agreement between self-reported and measured fitness was substantial (weighted $\kappa: 0.63$ ). High fitness patients were younger, frequently male, had a decreased prevalence of degenerative complications, lower office and ambulatory BP levels, particularly during the night and had an increased prevalence of the normal nocturnal dipping pattern. High fitness patients also had lower hemoglobin A1C and C-reactive protein levels, a decreased prevalence of left ventricular hypertrophy and increased aortic stiffness. On multiple logistic regression, after adjustments for several confounders, high fitness was independently associated with a higher likelihood of having low C-reactive protein (odds ratio (OR): $2.3,95 \%$ confidence interval (Cl): 1.3-3.9), controlled nighttime BP (OR: 2.3, 95\% Cl: 1.4-3.8), normal dipping pattern (OR: $2.1,95 \% \mathrm{Cl}: 1.2-3.5$ ) and low aortic stiffness (OR: $2.5,95 \% \mathrm{Cl}: 1.3-4.8$ ). Patients with moderate fitness had intermediate OR. In conclusion, a moderate to high level of physical fitness was independently associated with several favorable intermediate cardiovascular risk markers, which may contribute to decreasing the burden of morbi-mortality in patients with type 2 diabetes.

Hypertension Research (2011) 34, 856-861; doi:10.1038/hr.2011.50; published online 12 May 2011

Keywords: ambulatory blood pressure; aortic stiffness; cardiorespiratory fitness; C-reactive protein; type 2 diabetes

\section{INTRODUCTION}

Studies in type 2 diabetic patients have found that high levels of aerobic fitness are associated with lower levels of overall mortality and mortality due to cardiovascular events, independent of traditional cardiovascular risk factors. ${ }^{1,2}$ However, the relationship between cardiorespiratory fitness and some recently described non-traditional cardiovascular risk factors, such as ambulatory blood pressures (BPs), aortic stiffness and C-reactive protein, has not been as well characterized for type 2 diabetic patients. Whether these factors contribute independently to low fitness or merely reflect more advanced cardiovascular complications is unknown.

Compared with office BPs, ambulatory BPs are stronger cardiovascular risk predictors, ${ }^{3,4}$ and have stronger associations with targetorgan damage in type 2 diabetes. ${ }^{5}$ Studies using ambulatory BP monitoring (ABPM) suggest that endurance training may lower daytime BP in normal and hypertensive patients. ${ }^{6}$ The possible associations between cardiorespiratory fitness and ABPM parameters, and determination of whether these are independent of the presence of diabetic complications that can negatively impact exercise function have not yet been investigated. Besides, under debate is whether diabetic patients with lower fitness have increased aortic stiffness and, more importantly, whether physical activity can reduce arterial stiffness. ${ }^{7,8}$ Regarding C-reactive protein, some studies on type 2 diabetes reported associations with exercise capacity 9,10 and a reduction in C-reactive protein levels after a program of physical training. ${ }^{11,12}$

The aim of this study was to investigate the independent relationships between exercise capacity (evaluated by a self-reported questionnaire and, for a sub-sample of patients, by a standard treadmill test) and ambulatory BP monitoring data, aortic pulse wave velocity (PWV; which reflects arterial stiffness) and C-reactive protein, over and above other known conditions that negatively impact physical activity (such as the presence of chronic degenerative complications, obesity, arterial hypertension and left ventricular diastolic dysfunction).

\footnotetext{
${ }^{1}$ Department of Internal Medicine, University Hospital Clementino Fraga Filho, Medical School, Federal University of Rio de Janeiro, Rio de Janeiro, Brazil and ${ }^{2}$ Laboratory of Exercise Physiology, School of Physical Education and Sports, Federal University of Rio de Janeiro, Rio de Janeiro, Brazil

Correspondence: Dr GF Salles, Department of Internal Medicine, University Hospital Clementino Fraga Filho, Medical School, Federal University of Rio de Janeiro, Rua Croton 72 , Jacarepaguá, CEP: 22750-240 Rio de Janeiro, Brazil.

E-mail: gilsalles@hucff.ufr.br

Received 19 September 2010; revised 14 January 2011; accepted 9 February 2011; published online 12 May 2011
} 


\section{METHODS}

\section{Study patients and protocol}

This was a cross-sectional study within a cohort of 600 type 2 diabetic patients (38\% male, mean (s.d.) age: 60.4 (9.5) years) enrolled from August 2004 to December 2008 in the outpatient clinic of a tertiary care university hospital. Exclusion criteria to enter the cohort were a body mass index $>40 \mathrm{~kg} \mathrm{~m}^{-2}$, serum creatinine $\geqslant 180 \mu \mathrm{mol}^{-1}$ and the presence of any serious concomitant disease limiting life expectancy. ${ }^{13}$ For this study, 25 patients with severe walking disturbances because of previous strokes, severe intermittent claudication or severe knee osteoarthritis were also excluded. As a result, 575 patients were evaluated. All patients gave written informed consent, and the local ethics committee approved the study protocol. Patients were submitted to a standard protocol that included a complete clinical examination, a physical activity questionnaire, ${ }^{14}$ clinical tests of cardiovascular autonomic function, a laboratory evaluation, a 24-h ABPM, a two-dimensional echocardiogram and a carotid-femoral PWV measurement. ${ }^{15}$ Diagnostic criteria for macrovascular and microvascular degenerative complications and the protocol for cardiovascular autonomic function tests were previously described. ${ }^{13,15}$ Laboratory evaluation included fasting glycemia, serum creatinine and lipids, glycated hemoglobin, C-reactive protein and albuminuria. C-reactive protein was measured by a high-sensitivity immunonephelometry assay (lower detection limit: $0.1 \mathrm{mgl}^{-1}$, intra-assay and inter-assay variation coefficients: $3.1-4.0$ and $2.5-3.8 \%$, respectively); C-reactive protein measurement was repeated if the value of first dose was $>3 \mathrm{mgl}^{-1}$, and the lowest value was considered.

\section{Assessment of exercise capacity}

Each exercise capacity of the patient was estimated using a standard questionnaire, the Duke Activity Status Index, ${ }^{14}$ which assigns an estimated metabolic equivalent (MET) level for each type of activity reported on 12 questions. When necessary, this questionnaire was complemented by a more complete compendium of activities, ${ }^{16}$ which included more unusual activities such as work-related tasks, as applicable for some hard workers. In all, 265 randomly selected patients (46\%) also performed a maximum treadmill test, according to the Bruce protocol, and peak oxygen consumption $\left(\mathrm{VO}_{2 \mathrm{MAX}}\right)$ was measured with a respiratory gas analyzer (VO2000, MedGraphics, St Paul, MN, USA) on 50 of the patients undergoing the treadmill test to assess the accuracy of their self-reported cardiorespiratory fitness questionnaire estimations. We quantified exercise capacity as maximal METs by a formula commonly used for the Bruce protocol. ${ }^{17} \mathrm{~A}$ total of $80 \%$ of the patients achieved a maximum heart rate of at least $85 \%$ of the predicted heart rate. Each patient was grouped into one of the three categories according to estimated or measured physical capacity: low ( $<4$ METs), moderate ( $\geqslant 4$ to $<7$ METs) and high ( $\geqslant 7$ METs) fitness. These cutoff values divided patients in the $30 \%$ lowest, $40 \%$ in the middle-moderate and $30 \%$ in the highest fitness categories. All patients in the lowest fitness subgroup achieved a maximal heart rate of at least $85 \%$ of the predicted maximum heart rate, regardless of $\beta$-blocker use. Whenever there was disagreement between directly measured fitness and that estimated by the questionnaire, the treadmill test measurement defined the fitness category.

\section{BP, aortic PWV and echocardiographic measurements}

Office BP was measured three times using a digital oscillometric BP monitor (HEM-907 XL, Omron Healthcare, Kyoto, Japan) with a suitable sized cuff. The first measurement was discarded, and the reported BP was obtained by averaging the final two readings. Arterial hypertension was diagnosed when mean systolic BP exceeded $140 \mathrm{~mm} \mathrm{Hg}$ or diastolic BP was $>90 \mathrm{~mm} \mathrm{Hg}$ or if anti-hypertensive drugs had been previously prescribed. ABPM was recorded using Mobil O Graph version 12 equipment (Dynamapa, Cardios LTDA, São Paulo, Brazil). A reading was taken every $15 \mathrm{~min}$ throughout the day and every $30 \mathrm{~min}$ at night. The nighttime period for each individual patient was ascertained from registered diaries. The evaluated parameters were $24 \mathrm{~h}$, daytime and nighttime BPs. A normal circadian variability pattern (dipper) was defined as a nocturnal systolic BP fall $>10 \%$ in relation to daytime levels. A single independent observer measured carotid-femoral (aortic) PWV using the foot-to-foot velocity method with Complior equipment (Artech-Medical, Paris, France). ${ }^{15}$ Increased arterial stiffness was defined as aortic PWV $>12 \mathrm{~m} \mathrm{~s}^{-1}$. Two-dimensional transthoracic echocardiography (Sonoline
G60S, Siemens, Munich, Germany) was performed by the same experienced observer. Left ventricular mass was calculated and indexed to height ${ }^{2.7}$ (LVMI). Left ventricular hypertrophy was defined as LVMI $>44 \mathrm{~g} \mathrm{~m}^{-2.7}$ in women and $>48 \mathrm{~g} \mathrm{~m}^{-2.7}$ in men. Left ventricular diastolic function was also assessed using the mitral inflow Doppler pattern with standard criteria.

\section{Statistical analysis}

Statistics were performed with the SPSS 13.0 statistical package (SPSS, Chicago, IL, USA). Continuous variables were described as means and standard deviations when normally distributed and as medians and interquartile range when asymmetrically distributed. Agreement between grades of directly measured and estimated physical capacity was assessed by weighted $\kappa$-coefficient. One-way analysis of variance, Kruskal-Wallis or $\chi^{2}$-tests were used for overall comparisons between the three fitness subgroups. Post hoc Bonferroni's test (or $\chi^{2}$-test with Bonferroni's correction) was used for multiple comparisons between the subgroups, with the low fitness group as the reference. Independent associations of fitness grades with variables that reflect a better cardiovascular risk profile (low $\mathrm{C}$-reactive protein, controlled ambulatory nighttime $\mathrm{BP}$, normal dipping pattern, and low aortic stiffness) were examined by multivariate logistic regression, first in a crude non-adjusted model and then after full statistical adjustment for all potential confounders (age, gender, body mass index, diabetes duration, arterial hypertension and current anti-hypertensive treatment-number and class of anti-hypertensive drugs in use, dyslipidemia and statin use, presence of macro and microvascular complications, serum glycated hemoglobin and presence of left ventricular hypertrophy and diastolic dysfunction on echocardiogram). A subgroup analysis was also performed on those patients whose cardiorespiratory fitness was measured by the exercise treadmill test. Results were expressed as odds ratios (ORs) and $95 \%$ confidence intervals (CIs). A two-tailed $P$-value $<0.05$ was regarded as significant.

\section{RESULTS}

There was substantial agreement between physical capacity estimated by questionnaire and directly measured by treadmill test with $\mathrm{VO}_{2 \mathrm{MAX}}$ measurement (weighted $\kappa: 0.63)$. Overall, 184 patients (32\%) had low fitness, 221 (38\%) had moderate fitness and 170 (30\%) had high fitness. Tables 1 and 2 outline the characteristics of the three subgroups. The majority of the subgroup differences were observed between the high and the low fitness subgroups, and the moderate fitness subgroup tended to have intermediate values. Patients with high fitness were younger, frequently male, had lower body mass index and had a shorter diabetes duration. These patients had a lower prevalence of degenerative complications and arterial hypertension, used insulin less frequently and were taking fewer antihypertensive medications than patients with low fitness. Patients with high fitness had lower values of C-reactive protein and of mean glycated hemoglobin during the first year of follow-up. Office and ambulatory systolic BP, especially during the nighttime period, were lower in the high fitness subgroup; the high fitness group also had a higher prevalence of controlled BP levels and of a normal dipping pattern. They had lower values of LVMI and aortic PWV as well as a lower prevalence of left ventricular hypertrophy and of increased aortic stiffness (PWV $>12 \mathrm{~m} \mathrm{~s}^{-1}$ ).

The results of multivariate logistic regression analyses for the associations between fitness grades and markers of a better cardiovascular risk profile are shown in Table 3. There was a significant dose-response relationship (linear trend) between increasing physical fitness and all four markers of a better cardiovascular risk. After full statistical adjustment, high fitness diabetic subjects had a more than twofold increase in the odds of having a controlled nighttime BP $(<120 / 70 \mathrm{~mm} \mathrm{Hg})$, a normal dipping pattern, a low C-reactive protein level and normal aortic stiffness. Individuals with moderate fitness had a significantly increased chance of having a normal dipping 
Table 1 Baseline characteristics of patients according to fitness grades

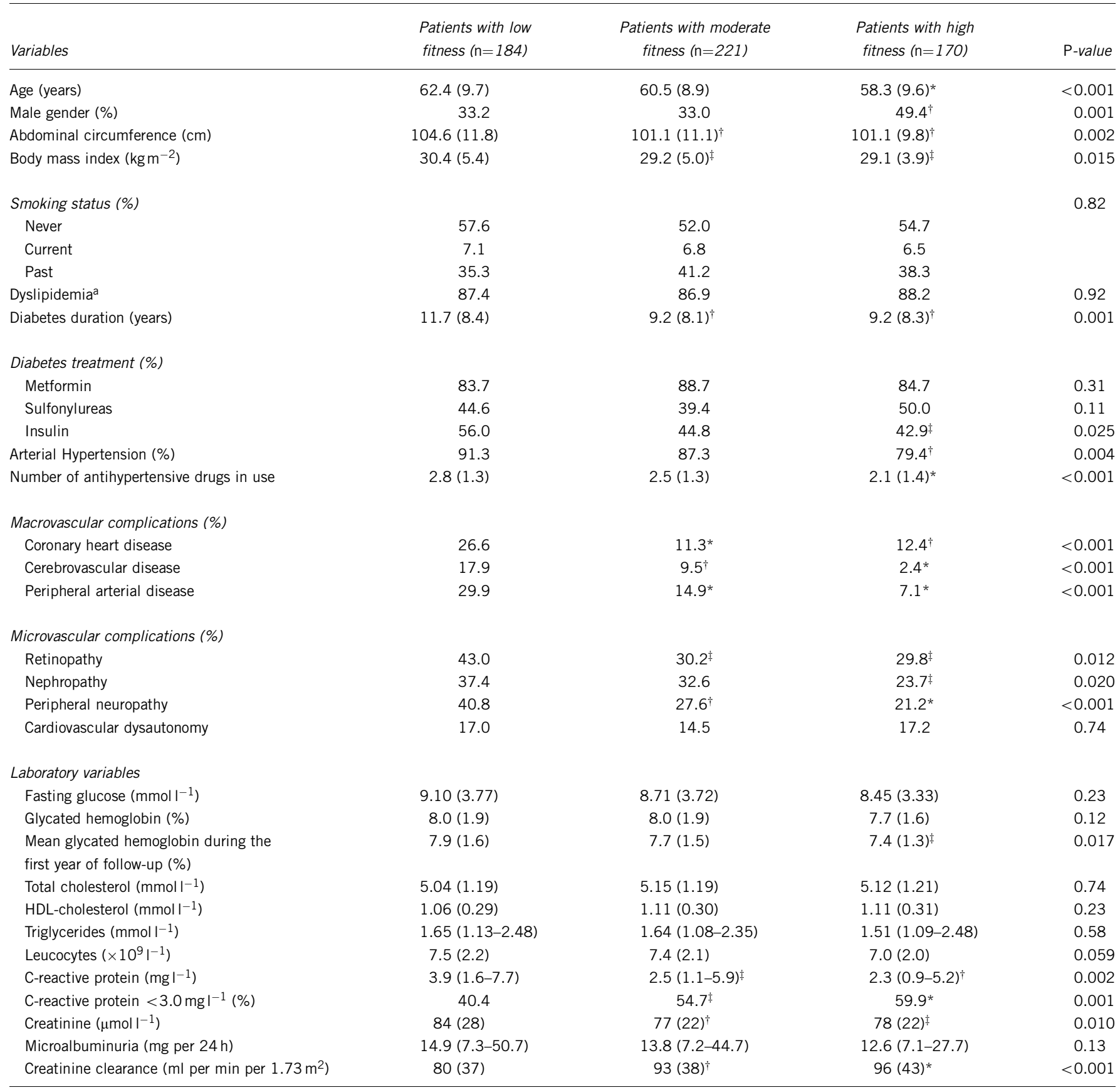

Abbreviation: HDL, high-density lipoprotein.

Values are means (standard deviations) for normally distributed continuous data, medians (interquartile range) for asymmetrically distributed continuous data and proportions for categorical data.

Values are means (standard deviations) for normally distributed continuous data, medians (interquartile range) for
$P$-values are for overall comparisons among all subgroups (by analysis of variance, Kruskal-Wallis or $\chi^{2}$-test).

$P$-values are for overall comparisons among all subgroups (by analysis of variance, Kruskal-Wallis or $\chi^{2}$-test).
${ }^{*} P<0.001,{ }^{\dagger} P<0.01,{ }^{\ddagger} P<0.05$ are for Bonferroni's post hoc comparisons with the reference category with low fitness.

aDislipidemia was defined by the National Cholesterol Education Program (Adult Treatment Panel III) criteria or by being prescribed any hypolipemic drug.

pattern and low C-reactive protein (increased by 1.7-fold and 1.9-fold, respectively) compared with low fitness patients. Analyses restricted to the 265 patients who performed exercise treadmill tests had nearly similar results to those observed in the entire study group, although some ORs lost statistical significance and some CIs became wider because of the smaller sample size. After full statistical adjustment of this analysis, highly fit individuals had 2.7 (95\% CI: 1.0-7.6, $P=0.05$ ), 3.0 (95\% CI: 1.1-8.2, $P=0.033$ ), 2.1 (95\% CI: $0.9-4.7, P=0.07)$ and 3.9 (95\% CI: $0.9-16.9, P=0.06$ ) increased odds of having controlled nighttime BP, a normal dipping pattern, a low C-reactive protein level and normal aortic stiffness, respectively.

\section{DISCUSSION}

This study investigated a large group of type 2 diabetic patients for relationships between cardiorespiratory fitness, which was evaluated both by a self-reported questionnaire (all patients) and by a standard treadmill test (sub-sample of patients), and four relatively novel cardiovascular risk markers: ABPM parameters (nighttime BP levels 
Table 2 Office and ambulatory blood pressures, aortic stiffness and echocardiographic variables in patients grouped according to fitness grades

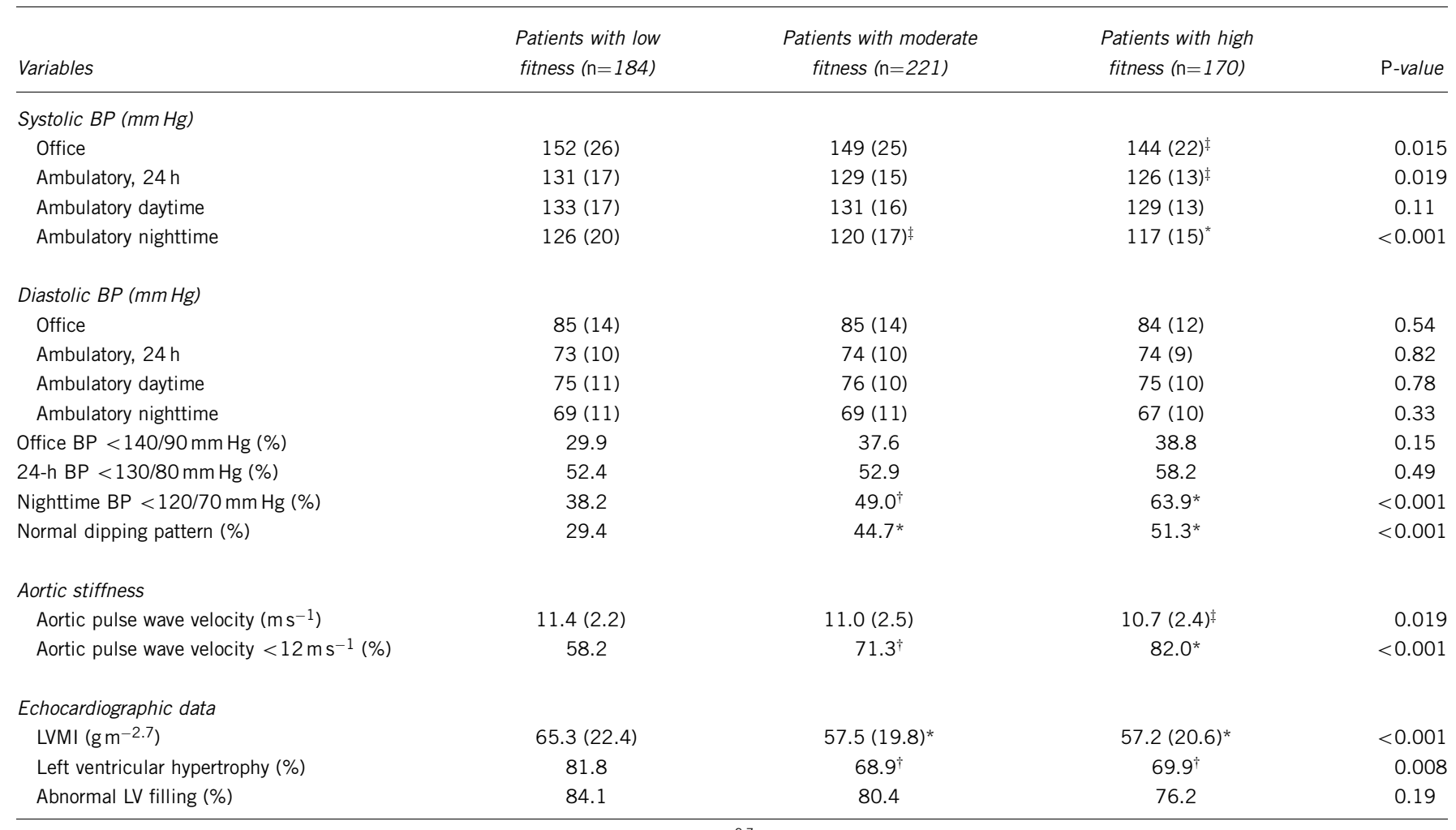

Abbreviations: BP, blood pressure; LV, left ventricular; LVMI, left ventricular mass indexed to height ${ }^{2.7}$

Values are means (standard deviations) or proportions.

$P$-values are for overall comparisons among all subgroups (by analysis of variance or $\chi^{2}$ test).

${ }^{*} P<0.001,{ }^{\dagger} P<0.01,{ }^{\ddagger} P<0.05$ are for Bonferroni's post hoc comparisons with the reference category with low fitness.

and dipping pattern), aortic stiffness and C-reactive protein. The study demonstrated that diabetic patients with moderate-high fitness have a better cardiovascular risk profile than those with low fitness and, more importantly, that these relationships are independent of conditions that can potentially impact exercise capacity, such as age, gender, obesity, smoking, diabetes duration, presence of microvascular and macrovascular complications such as cardiovascular autonomic neuropathy, use of anti-hypertensive medications and statins, and echocardiographic left ventricular diastolic dysfunction.

In this study, we demonstrated an inverse independent association between cardiorespiratory fitness and C-reactive protein levels. This finding is in accordance with the relationships found between C-reactive protein levels and self-reported physical activity in healthy multiethnic populations. ${ }^{18}$ Studies of type 2 diabetic patients also support these findings. A study of 28 female patients reported that there is an inverse correlation between fitness and C-reactive protein level. ${ }^{9}$ Another study of 425 men found in a multivariate linear regression with $\mathrm{C}$-reactive protein as the dependent variable an inverse relation with peak oxygen uptake and a direct relation with percent body fat, systolic BP and heart rate. ${ }^{10}$ Furthermore, small studies of diabetic patients demonstrated reductions in C-reactive protein levels in the exercise intervention subgroup. ${ }^{11,12}$ However, for type 2 diabetes, the association between C-reactive protein and development of cardiovascular disease is still under debate. Moreover, unknown is the extent to which the beneficial combined effects of exercise on metabolism, inflammation and vascular function can be expected to reduce the prevalence of atherosclerotic disease in diabetic patients.

We found that patients with high fitness have lower aortic stiffness, measured by the gold standard carotid-femoral PWV, than those with low fitness. This finding adds significant cardiovascular risk to low fitness patients because diabetic patients with increased aortic stiffness have a higher risk of cardiovascular events. ${ }^{19}$ Nonetheless, a correlation between cardiorespiratory fitness and aortic stiffness has not been consistently demonstrated. ${ }^{9}$ Diabetes has been shown to accelerate age-associated arterial stiffening, mainly through non-enzymatic glycation, which leads to the formation of increased collagen crosslinks that result in increased arterial stiffness. ${ }^{20}$ It has been hypothesized that pulsatile stretching of collagen fibers during aerobic exercise could break the collagen links and result in decreased arterial stiffness. ${ }^{21}$ However, the impact of aerobic exercise on arterial stiffness in diabetic patients is still uncertain. A 3-month study with 36 elderly diabetic patients randomized to aerobic or anaerobic exercise showed a reduction in arterial stiffness in the aerobic group, ${ }^{8}$ whereas another study with 50 patients randomized to exercise training or standard treatment did not show any change in aortic stiffness after a 24-month program. ${ }^{7}$

The observed independent association between high fitness and a better ABPM profile, particularly during the nighttime period, identifies patients with a better cardiovascular risk profile. Nighttime BP levels and dipping pattern consistently have been demonstrated to predict better cardiovascular prognosis than office and daytime BPs in population-based studies ${ }^{3}$ and in hypertensive individuals. ${ }^{22}$ However, reports on this issue in type 2 diabetes are scarce. ${ }^{4,23}$ Studies assessing the prognostic value of ABPM parameters in type 2 diabetic patients have been limited to White and Japanese populations, and these studies had methodological limitations, such as relatively small sample sizes and a small number of events, ${ }^{4,23,24}$ interfering with a more comprehensive multivariate analysis. Another study with elderly 
diabetic patients failed to demonstrate any prognostic importance of ambulatory BPs after multivariate adjustment. ${ }^{25}$ A meta-analysis of randomized controlled trials showed that aerobic training indeed lowers office and ambulatory BPs, and the net effect is more pronounced in hypertensive than in non-hypertensive individuals. ${ }^{6}$ The pathophysiological mechanisms underlying exercise-induced BP reduction seem to be the result of a decrease in systemic vascular resistance, and attenuation of the sympathetic nervous and reninangiotensin systems appears to be involved. ${ }^{6}$ In addition, a reduction in insulin resistance and the improvement of endothelial dysfunction are other potentially important mechanisms that may contribute to the favorable effect of endurance exercise on $\mathrm{BP}^{26}$

Some limitations of this study should be mentioned. First, the cross-sectional design of this study precludes any causal inferences regarding the associations between high cardiorespiratory fitness and the novel cardiovascular risk markers. Second, cardiorespiratory fitness was self-reported in $54 \%$ of the patients and may have been overestimated because obese subjects tend to over-report their exercise capacity. ${ }^{27}$ However, we demonstrated a substantial correlation between self-reported fitness and that directly measured by $\mathrm{VO}_{2 \mathrm{MAX}}$ in a random subgroup of patients on a standard treadmill test. In addition, self-reported exercise habits correlate with physical capacity and can be used to infer fitness with decent accuracy. ${ }^{14,28,29}$ The strength of the associations between high fitness and cardiovascular risk markers would be attenuated by overestimation of cardiorespiratory fitness and the resulting misclassification of patients into the moderate or high fitness group when they were actually low or moderate fitness individuals, respectively. Hence, the relationships found here should be considered conservative estimates; the true associations may be even stronger. Third, inter-observer reproducibility assessment could not be performed for the PWV and echocardiographic parameters because there was only a single observer measurement for these parameters. Finally, this study was conducted in a tertiary care hospital with a patient population of predominantly middle aged to elderly individuals; thus, our findings may not be generalizable to younger diabetic patients and to patients followed at primary care centers.

In conclusion, we demonstrated that a high exercise capacity is independently associated with novel cardiovascular risk markers. The strength of the study is the fact that the associations remain independent after adjustment for conditions that can negatively impact exercise capacity. In perspective, the extent to which the combined beneficial effects of exercise on metabolism and on traditional and less conventional risk factors, such as ABPM parameters, aortic stiffness and C-reactive protein, can be expected to reduce atherosclerotic cardiovascular disease remains unclear and should be investigated in future interventional, prospective studies.

\section{ACKNOWLEDGEMENTS}

The study was supported by research grants from Conselho Brasileiro de Desenvolvimento Científico e Tecnológico (CNPq) and from Fundação Carlos Chagas Filho de Amparo à Pesquisa do Estado do Rio de Janeiro (FAPERJ).

1 Church TS, Cheng YJ, Earnest CP, Barlow CE, Gibbons LW, Priest EL, Blair SN. Exercise capacity and body composition as predictors of mortality among men with diabetes. Diabetes Care 2004; 27: 83-88.

2 Kokkinos P, Myers J, Nylen E, Panagiotakos DB, Manolis A, Pittaras A, Blackman MR, Jacob-Issac R, Faselis C, Abella J, Singh S. Exercise capacity and all-cause mortality in African American and Caucasian men with type 2 diabetes. Diabetes Care 2009; 32 : 623-628. 
3 Boggia J, Li Y, Thijs L, Kikuya M, Björklund-Bodegård K, Richart T, Ohkubo T, Kuznetsova T, Torp-Pedersen C, Lind L, Ibsen H, Imai Y, Wang J, Sandoya E, O'Brien $\mathrm{E}$, Staessen JA, International Database on Ambulatory blood pressure monitoring in relation to Cardiovascular Outcomes (IDACO) investigators. Prognostic accuracy of day versus night ambulatory blood pressure: a cohort study. Lancet 2007; 370: 1219-1229.

4 Eguchi K, Pickering TG, Hoshide S, Ishikawa J, Ishikawa S, Schwartz JE, Shimada K, Kario K. Ambulatory blood pressure is a better marker than clinic blood pressure in predicting cardiovascular events in patients with/without type 2 diabetes. Am J Hypertens 2008; 21: 443-450.

5 Knudsen ST, Laugesen E, Hansen KW, Bek T, Mogensen CE, Poulsen PL. Ambulatory pulse pressure, decreased nocturnal blood pressure reduction and progression of nephropathy in type 2 diabetic patients. Diabetologia 2009; 52: 698-704.

6 Cornelissen VA, Fagard RH. Effects of endurance training on blood pressure, blood pressure-regulating mechanisms, and cardiovascular risk factors. Hypertension 2005; 46: 667-675.

7 Loimaala A, Groundstroem K, Rinne M, Nenonen A, Huhtala H, Parkkari J, Vuori I. Effect of long-term endurance and strength training on metabolic control and arterial elasticity in patients with type 2 diabetes mellitus. Am J Cardiol 2009; 103: 972-977.

8 Madden KM, Lockhart C, Cuff D, Potter TF, Meneilly GS. Short-term aerobic exercise reduces arterial stiffness in older adults with type 2 diabetes, hypertension, and hypercholesterolemia. Diabetes Care 2009; 32: 1531-1535.

9 McGavock JM, Mandic S, Vonder Muhll I, Lewanczuk RZ, Quinney HA, Taylor DA, Welsh RC, Haykowsky M. Low cardiorespiratory fitness is associated with elevated C-reactive protein levels in women with type 2 diabetes. Diabetes Care 2004; 27: 320-325.

10 Jae SY, Heffernan KS, Lee MK, Fernhall B, Park WH. Relation of cardiorespiratory fitness to inflammatory markers, fibrinolytic factors, and lipoprotein (a) in patients with type 2 diabetes mellitus. Am J Cardiol 2008; 102: 700-703.

11 Oberbach A, Tönjes A, Klöting N, Fasshauer M, Kratzsch J, Busse MW, Paschke R, Stumvoll M, Blüher M. Effect of a 4 week physical training program on plasma concentrations of inflammatory markers in patients with abnormal glucose tolerance. Eur J Endocrinol 2006; 154: 577-585.

12 Kadoglou NP, Iliadis F, Angelopoulou N, Perrea D, Ampatzidis G, Liapis CD, Alevizos M. The anti-inflammatory effects of exercise training in patients with type 2 diabetes mellitus. Eur J Cardiovasc Prev Rehabil 2007; 14: 837-843.

13 Cardoso CR, Leite NC, Freitas L, Dias SB, Muxfeld ES, Salles GF. Pattern of 24-h ambulatory blood pressure monitoring in type 2 diabetic patients with cardiovascular dysautonomy. Hypertens Res 2008; 31: 865-872.

14 Hlatky MA, Boineau RE, Higginbotham MB, Lee KL, Mark DB, Califf RM, Cobb FR, Pryor DB. A brief self-administered questionnaire to determine functional capacity (the Duke Activity Status Index). Am J Cardiol 1989; 64: 651-654.

15 Cardoso CR, Ferreira MT, Leite NC, Barros PN, Conte PH, Salles GF. Microvascular degenerative complications are associated with increased aortic stiffness in type 2 diabetic patients. Atherosclerosis 2009; 205: 472-476.
16 Ainsworth BE, Haskell WL, Whitt MC, Irwin ML, Swartz AM, Strath SJ, O'Brien WL, Bassett Jr DR, Schmitz KH, Emplaincourt PO, Jacobs Jr DR, Leon AS. Compendium of physical activities: an update of activity codes and MET intensities. Med Sci Sports Exerc 2000; 32(Suppl): S498-S504.

17 Foster C, Jackson AS, Pollock ML, Taylor MM, Hare J, Sennett SM, Rod JL, Sarwar M, Schmidt $\mathrm{DH}$. Generalized equations for predicting functional capacity from treadmill performance. Am Heart J 1984; 107: 1229-1234.

18 Geffken DF, Cushman M, Burke GL, Polak JF, Sakkinen PA, Tracy RP. Association between physical activity and markers of inflammation in a healthy elderly population. Am J Epidemiol 2001; 153: 242-250.

19 Cruickshank K, Riste L, Anderson SG, Wright JS, Dunn G, Gosling RG. Aortic pulse wave velocity and its relationship to mortality in diabetes and glucose intolerance: an integrated index of vascular function? Circulation 2002; 106: 2085-2090.

20 Lakatta EG. Arterial and cardiac aging: major shareholders in cardiovascular disease enterprises: Part III: cellular and molecular clues to heart and arterial aging. Circulation 2003; 107: 490-497.

21 Cencetti S, Lagi A, Cipriani M, Fattorini L, Bandinelli G, Bernardi L. Autonomic control of the cerebral circulation during normal and impaired peripheral circulatory control. Heart 1999; 82: 365-372.

22 Muxfeldt ES, Cardoso CR, Salles GF. Prognostic value of nocturnal blood pressure reduction in resistant hypertension. Arch Intern Med 2009; 169: 874-880.

23 Nakano S, Ito T, Furuya K, Tsuda S, Konishi K, Nishizawa M, Nakagawa A, Kigoshi T, Uchida K. Ambulatory blood pressure level rather than dipper/nondipper status predicts vascular events in type 2 diabetic subjects. Hypertens Res 2004; 27: 647-656.

24 Astrup AS, Nielsen FS, Rossing P, Ali S, Kastrup J, Smidt UM, Parving HH. Predictors of mortality in patients with type 2 diabetes with or without diabetic nephropathy: a follow-up study. J Hypertens 2007; 25: 2479-2485.

25 Palmas W, Pickering TG, Teresi J, Schwartz JE, Moran A, Weinstock RS, Shea S. Ambulatory blood pressure monitoring and all-cause mortality in elderly people with diabetes mellitus. Hypertension 2009; 53: 120-127.

26 Higashi Y, Sasaki S, Kurisu S, Yoshimizu A, Sasaki N, Matsuura H, Kajiyama G, Oshima T. Regular aerobic exercise augments endothelium-dependent vascular relaxation in normotensive as well as hypertensive subjects: role of endothelium-derived nitric oxide. Circulation 1999; 100: 1194-1202.

27 Lichtman SW, Pisarska K, Berman ER, Pestone M, Dowling H, Offenbacher E, Weisel $H$, Heshka S, Matthews DE, Heymsfield SB. Discrepancy between self-reported and actual caloric intake and exercise in obese subjects. New Engl J Med 1992; 327: 1893-1898.

28 Jackson AS, Blair SN, Mahar MT, Wier LT, Ross RM, Stuteville JE. Prediction of functional aerobic capacity without exercise testing. Med Sci Sports Exerc 1990; 22: 863-870.

29 Jurca R, Jackson AS, LaMonte MJ, Morrow Jr JR, Blair SN, Wareham NJ, Haskell WL, van Mechelen W, Church TS, Jakicic JM, Laukkanen R. Assessing cardiorespiratory fitness without performing exercise testing. Am J Prev Med 2005; 29: 185-193. 